\section{P5.06 HIV-POSITIVE MEN'S KNOWLEDGE AND ATTITUDES REGARDING HPV, HPV VACCINE, AND ANAL CANCER SCREENING}

${ }^{1}$ Ann Burchell, ${ }^{2}$ Gina Ogilvie, ${ }^{1}$ Ramandip Grewal, ${ }^{3}$ Janet Raboud, ${ }^{4}$ Troy Grennan, ${ }^{3}$ Irving Salit. 'St. Michael's Hospital, Canada; ${ }^{2}$ University of British Columbia, Canada; ${ }^{3}$ University Health Network, Canada; ${ }^{4}$ British Columbia Centre for Disease Control, Canada

\subsection{6/sextrans-2017-053264.622}

Introduction High-risk oncogenic types of human papillomavirus (HPV) are the major cause of several cancers in men, notably anal cancer. HIV co-infection further elevates risk. We assessed knowledge of and attitudes regarding HPV, HPV vaccination, anal cancer, and anal cancer screening among HIVpositive men.

Methods Male participants of a multi-site cohort of persons in care at 9 specialty HIV clinics in Ontario, Canada, responded to questions about HPV knowledge and attitudes regarding perceived risk and willingness to receive HPV vaccine and anal cancer screening. We analysed data from interviews between 04/2016 and 08/2016.

Results 678 men were interviewed. Many had either not heard of HPV $(20 \%)$ or had heard the term but did not know what it was $(25 \%)$. Among men familiar with the term $(n=398)$, only $51 \%$ knew that HPV can cause anal cancer and $56 \%$ knew that people with HIV are at higher risk for cancers caused by HPV. Many thought their chance of getting HPV was zero (19\%) or low (36\%). 63\% had heard of the HPV vaccine and $44 \%$ knew that it was recommended for males, but only $13 \%$ reported that a health professional discussed the vaccine with them and $6 \%$ were vaccinated. Men said that they would be likely/very likely to get vaccinated if it were offered free of charge (81\%), if they had to co-pay $\$ 30$ / dose $(59 \%)$, or pay full price $(18 \%)$. The majority would be likely/very likely to get anal cancer screening via digital rectal exam (87\%), Pap cytology (90\%), or anoscopy (83\%). Men were comfortable/very comfortable discussing anal health with their HIV doctor (87\%) and family doctor (84\%).

Conclusion Our findings suggest that there are many knowledge gaps to address regarding awareness of HPV and HPVassociated disease among HIV-positive men, but that men were comfortable discussing anal health with care providers. Men were generally willing to accept anal cancer screening and HPV vaccination, if offered at no/low cost. Future work will identify correlates of hesitancy to accept vaccination and screening, which can guide promotional messaging and interventions.

\section{P5.07 PRACTICABILITY EVALUATION IN FRENCH-SPEAKING SUB-SAHARAN AFRICA COUNTRIES OF FINGER-STICK WHOLE BLOOD HIV SELF-TEST}

${ }^{1}$ Belec, ${ }^{2}$ Serge Tonen Wolyec, ${ }^{3}$ Gérard Grésenguet. ${ }^{1}$ Paris V University, France; ${ }^{2}$ Université de Bunia; ${ }^{3}$ Université de Bangui

\subsection{6/sextrans-2017-053264.623}

Introduction Opportunities for HIV testing could be enhanced by offering HIV self-testing (HIVST) in populations that fear stigma and discrimination when accessing conventional HIV counselling and testing in health care facilities.

Methods The Exacto Test HIV (Biosynex, Strasbourg, France) fullfilled the following criteria I) Capillary blood-based test detecting early HIV infection; II) Sterile safety lancet; III)
Simplified blood sampling system; IV) Simplified buffer delivery system; V) Specimen presence control by blood deposit assessment and migration control band; vi) Results in $10 \mathrm{~min}$; VII) Simplified and easy-to-read leaflet in French, Lingala, Swahili and Sango. The practicability of HIVST was assessed in 630 adults living in Kisangani and Bunia, Democratic Republic of Congo $(n=330)$ and Bangui, Central African Repulic ( $\mathrm{n}=300)$, according to WHO recommendations.

Results Design. Exacto self-test showed 100.0\% sensitivity and 99.5\% specificity. Practicability in lay users. $97.4 \%$ correctly performed HIVST and claimed the test was easy or very easy to carry out; however, $26.5 \%$ asked for telephone or oral assistance, and difficulty obtaining sufficient quantity of blood was observed in $2.6 \% ; 47 \%$ used the notice in French, $10 \%$ in Lingala, 23\% in Swahili and 20\% in Sango. Overall, the results were correctly interpreted in $90.2 \%$, the reading/interpretation errors concerned the positive (6.5\%), negative $(11.2 \%)$ or invalid $(16.0 \%)$ self-tests. The notice was correctly understood in $78.5 \%$.

Conclusion Our field experience with Exacto self-test implemented for the first time in a French-speaking African country demonstrates very satisfactrory success rate of performance and interpretation. However, the main obstacle for HIVST was clearly the educational level, associated with execution and interpretation difficulties in illeterates or very poorly educated people. Notice design with pictures and in vernacular languages is essential. Supervised use of HIVST should be essential in poorly-educated people.

\section{P5.08 KNOWLEDGE OF TRUVADA PRE EXPOSURE PROPHYLAXIS (PREP) AMONG MEN WHO HAVE SEX WITH MEN IN KISUMU COUNTY, KENYA}

Charles Kiplagat. Kenyatta National Hospital, Kenya

\subsection{6/sextrans-2017-053264.624}

Introduction Each country in the world has put in place strategies to prevent HIV/AIDS infections. In Kenya today, to control HIV/AIDS infection, there are various strategies that are in place: VCT,PMTCT,VMMC and Condom use. In that context however, research has shown that Truvada PrEP reduces the risk of HIV infection by more than 90\% among gay and bisexual men when taken once-daily The objective of this study is to investigate the knowledge of Pre Exposure Prophylaxis (PreP) among MSM.

Method Ten community health workers were subjected to a three week intensive training programs to empower them with skills of carrying out interviews. Data was collected for a period of three months by use of Questionnaires and personal interviews. During the study, the enumerators used the following demographic data: Sex, Age, Education and how the partners perceive the use of Pre Exposure Prophylaxis

Results 80 MSM were enrolled in this survey with their ages ranging from 18-35 years all being Males who are HIV + and with basic High Education. Of $40 \%$ of the respondents, they have preferred to use condoms as a protective device and other Gels other than Truvada PreP.50\% of the respondents were in agreement to use PreP and they had some perceptions on the cost, availability, side effects in the event of stoppage while $10 \%$ of the respondents were not sure whether to use Truvada PreP or not since they needed more 